\title{
Identificação e análise das diretrizes nacionais de atividade física nas Américas
}

\author{
Identification and analysis of national physical activity guidelines \\ in the Americas
}

Paulo Henrique Guerra (https://orcid.org/0000-0003-4239-0716) 1,2

Leandro Martin Totaro Garcia (https://orcid.org/0000-0001-5947-2617) ${ }^{2}$

Evelyn Helena Corgosinho Ribeiro (https://orcid.org/0000-0002-3565-6830) ${ }^{2}$

Ana Lúcia Rodrigues Maretti (https://orcid.org/0000-0001-8193-4128) ${ }^{2}$

Douglas Roque Andrade (https://orcid.org/0000-0001-5135-582X) ${ }^{2}$

Alex Antonio Florindo (https://orcid.org/0000-0002-4429-0826) ${ }^{2}$
${ }^{1}$ Universidade Federal da Fronteira Sul. Bairro Fronteira Sul s/n Km 02 Bloco dos professores sala 110, Fronteira Sul. 89802112 Chapecó SC Brasil. paulo.guerra@uffs.edu.br ${ }^{2}$ Grupo de Estudos e Pesquisas Epidemiológicas em Atividade Física e Saúde, Universidade de São Paulo. São Paulo SP Brasil.

\begin{abstract}
Given the potential of institutional approaches to health promotion, this study sought to identify the countries on the American continent which have national physical activity (PA) guidelines approved by their national health agencies. A scoping review was conducted in May 2019, with a search conducted in five electronic databases, national health agency websites and contacts with members of the Physical Activity Network of the Americas. In all, national PA guidelines were found in ten of the 36 countries surveyed (27.8\%), published between 2011 and 2018, the majority being in South American countries $(n=6)$. All guidelines presented "approaches and strategies for primary health care," "PA recommendations for health," and the "involvement of different PA domains," however, few guidelines addressed "constructed environment and infrastructure" ( $n$ =3), as well as "monitoring and surveillance actions" $(n=5)$. The conclusion can be drawn that, although few countries on the American continent have guidelines for $P A$ and that the majority of those available address $P A$ in a limited way, new national guidelines, based on the complexity of the determinants, monitoring and consequences of $P A$ are needed to guide and foster policies to promote $P A$ within the population.
\end{abstract}

Key words Physical activity, Public policy, Health promotion
Resumo Visto o potencial das abordagens institucionais à promoção da saúde, o presente estudo buscou identificar os países do continente americano que possuem diretrizes nacionais de atividade fisica (AF) chanceladas por órgãos nacionais de saúde. Em maio de 2019 realizou-se uma revisão de escopo, com buscas em cinco bases de dados eletrônicas, sitios eletrônicos dos órgãos nacionais de saúde e contato com integrantes da Rede de Atividade Física das Américas. Foram encontradas diretrizes nacionais de AF em dez dos 36 países pesquisados (27,8\%), publicadas entre 2011 e 2018, com maioria nos países da América do Sul ( $n=6)$. Todas as diretrizes apresentaram "abordagens e estratégias para a atenção primária em saúde", "recomendações de AF para a saúde" e o "envolvimento de distintos domínios da AF", contudo, poucas diretrizes abordaram o "ambiente construído e infraestrutura" ( $n=3)$, assim como "ações de monitoramento e vigilância" $(n=5)$. Pode-se concluir que, ao passo que poucos países do continente americano dispõem de diretrizes para a $A F$ e, que boa parte das disponiveis aborda a $A F$ de maneira limitada, novas diretrizes nacionais, fundamentadas na complexidade dos determinantes, monitoramento e consequências da $A F$ são necessárias para a orientação e incentivo de politicas de promoção da AF no contexto populacional. Palavras-chave Atividade física, Politica pública, Promoção da saúde 


\section{Introdução}

Em reconhecimento dos inúmeros benefícios à saúde ao longo da vida, a atividade física $(\mathrm{AF})$ é recomendada pela Organização Mundial da Saúde (OMS) como um dos principais determinantes de saúde pública ${ }^{1,2}$. Mesmo assim, em vista das elevadas prevalências de pessoas inativas ao redor do mundo ${ }^{3}$, de acordo com as recomendações de AF moderadas e vigorosas (150 minutos semanais de AF moderadas ou 75 minutos semanais de $\mathrm{AF}$ vigorosas) ${ }^{4}$, a OMS também recomenda a implementação de diversas estratégias para a sua promoção no nível populacional, como, por exemplo, a formulação e atualização periódica de diretrizes nacionais de AF.

No contexto ampliado da saúde, estas diretrizes, fundamentadas em evidências científicas e geralmente elaboradas por painéis de experts na temática, têm como objetivo orientar políticas públicas, incentivar a população em geral e informar a atuação dos profissionais para a adoção de hábitos saudáveis ${ }^{5}$. Estas diretrizes podem também direcionar estratégias e ações no contexto dos sistemas nacionais para além do setor saúde (exemplo educação, esporte e transporte), sendo, dessa forma, um instrumento de grande valia para articulações intersetoriais e interprofissionais.

Ao se analisar como os países no mundo estão procedendo em relação à formulação de diretrizes nacionais de AF, pode-se destacar o levantamento europeu onde, dos 53 países investigados, foram encontradas 21 recomendações nacionais de AF chanceladas por órgãos governamentais ${ }^{6}$. Além disso, estudo da segunda série Lancet de AF mostrou que vem aumentando o número de países com políticas, planos de ações e estratégias de promoção da atividade física no mundo ${ }^{3}$. Mesmo que estes estudos tenham apontado o aumento do número de países que possuem recomendações nacionais de AF na Europa e no mundo em um curto espaço de tempo ${ }^{3,7}$, também apontam que boa parcela destas orientações está alicerçada na recomendação estadunidense de 1995 (acumular 30 minutos ou mais de AF de intensidade moderada na maioria dos dias da semana, preferencialmente em todos $)^{8}$ e não na recomendação de 2010 da $\mathrm{OMS}^{4}$, que era a mais atual na época de publicação do estudo. Ainda, diretrizes elaboradas mais recentemente ao redor do mundo têm adotado outras abordagens e mensagens, por exemplo, dedicando-se às atividades nas 24 horas diárias e incluindo o tempo sentado e o sono ${ }^{9,10}$.

Diante da inexistência de trabalhos que analisaram de forma aprofundada as diretrizes de $\mathrm{AF}$ existentes nos países do continente americano e da importância de se fazê-lo em vista dos distintos benefícios à saúde da $\mathrm{AF}$ e do papel que as diretrizes podem desempenhar na promoção de um modo de vida mais ativo, os objetivos do presente trabalho foram: identificar os países do continente americano que possuem diretrizes nacionais de $\mathrm{AF}$ e analisar as informações das diretrizes nacionais disponíveis.

\section{Métodos}

O presente trabalho se trata de uma revisão de escopo. Foram consideradas para a síntese as diretrizes nacionais como guias e planos de AF chanceladas pelos órgãos nacionais de saúde dos 36 países que compõem o continente americano (https://nacoesunidas.org/conheca/paises-membros /\#paisesMembros1): Canadá, Estados Unidos, México, Antígua e Barbuda, Bahamas, Barbados, Belize, Costa Rica, Cuba, Dominica, El Salvador, Granada, Guatemala, Haiti, Honduras, Jamaica, Nicarágua, Panamá, República Dominicana, Santa Lúcia, São Cristóvão e Névis, São Tomé e Príncipe, São Vicente e Granadinas, Trinidad e Tobago, Argentina, Bolívia, Brasil, Chile, Colômbia, Equador, Guiana, Paraguai, Peru, Suriname, Uruguai e Venezuela. A opção pela chancela do setor saúde, especificamente, se deu em vista da liderança deste setor na elaboração dos guias nacionais ${ }^{6}$.

Em relação aos documentos, não se estipulou previamente nenhum tipo de restrição em relação aos seus propósitos, público-alvo, faixa etária, estratégias e intencionalidades, grupos populacionais abrangidos, tipos, intensidades e domínios de AF. Com o reconhecimento de que alguns órgãos nacionais de saúde apresentavam mais de uma diretriz nacional de AF, independentemente se para complementar a diretriz principal, ou mesmo direcionar orientações a grupos mais específicos, para os fins da síntese todas estas diretrizes também foram incluídas.

Por outro lado, não foram incluídos documentos como políticas (sem a característica de uma diretriz, como, por exemplo, envolvimento de tópicos sobre "como" fazer ou mesmo "orientar" AF), leis e panfletos (flyers e folders) apontando a importânciada prática de AF. Também, por se considerar a necessidade de revisão e atualização constante das diretrizes, em vista do desenvolvimento científico na temática da AF para a saúde, estipulou-se previamente que seriam incluídas as diretrizes nacionais publicadas a partir 
de 2005, considerando sempre sua edição mais atual, no caso dos países que ao longo do tempo promoveram atualizações em suas diretrizes. Esta opção se deu em vista do impacto deste tipo de documento no nível populacional e, também, pelo processo contínuo de descobertas científicas na temática, percebendo-se a necessidade de atualização periódica destes documentos.

Três estratégias foram desenvolvidas para a obtenção das diretrizes: 1) dois pesquisadores (ER, PG), de forma independente, aplicaram buscas sistemáticas em cinco bases de dados eletrônicas (Physical Education Index, PubMed, Scielo, Sport Discus e Web of Science), tomando como base os termos "guide*", "recommendations", "physicalactivity", "energy expenditure". $\mathrm{Na}$ base de dados SciElo as buscas também foram aplicadas nos idiomas espanhol e português; 2) realização de pesquisas nos sítios eletrônicos dos 36 órgãos nacionais de saúde dos países americanos ( $\mathrm{AF}, \mathrm{AR}, \mathrm{DA}, \mathrm{ER}, \mathrm{LG}, \mathrm{PG}$ ), assim como uma busca na página eletrônica do Google pelos próprios guias, partindo-se dos termos aplicados nas bases de dados, além do nome do país (considerando-se as grafias nos respectivos idiomas locais);e 3) a realização de contatos, via e-mail (DA, LG, PG), aos integrantes da Rede de Atividade Física das Américas (RAFA/PANA; https:// rafapana.org/), questionando sobre a existência e, nos casos positivos, solicitando os documentos locais. O rastreamento pelas diretrizes, nas bases de dados e nas páginas eletrônicas dos órgãos nacionais de saúde, foi realizado até maio de 2019.

Em posse de todas as diretrizes recuperadas, foi realizada uma leitura inicial dos documentos, em duplicidade e de forma independente (LG, PG), no sentido de identificar se estas respondiam aos principais pontos de inclusão (exemplo, chancela do órgão nacional de saúde e ano de publicação). Nas diretrizes que responderam adequadamente aos critérios supracitados, realizou-se a exploração do material, elencando-se elementos e abordagens utilizadas (exemplo, presença de recomendações de AF, tipos e contextos para a prática de $\mathrm{AF}$, direcionamentos para a promoção de AF). Os processos de exploração, extração e interpretação dos dados, tal como de construção das sínteses foram conduzidos pelos mesmos pesquisadores, também de forma independente (LG, PG). Em casos de desacordo durante a extração, os consensos foram alcançados por meio de reuniões.

\section{Resultados}

Ao todo, foram encontradas dez diretrizes nacionais de AF chanceladas pelos seus respectivos órgãos nacionais de saúde (10/36;27,8\%): Argentina $^{11}$, Canadá ${ }^{10}$, Chile ${ }^{12-14}$, Colômbia ${ }^{15-18}$, Costa Rica ${ }^{19}$, Equador, Estados Unidos da América (EUA) $^{21,22}$, Jamaica ${ }^{23,24}$, Peru ${ }^{25}$ e Uruguai ${ }^{26}$ (Quadro 1). A maior parte das diretrizes foi recuperada pela busca nos sítios eletrônicos dos órgãos nacionais de saúde, posteriormente corroboradas pelas respostas dos componentes da RAFA/ PANA. Por subdivisões do continente, seis diretrizes nacionais são oriundas da América do Sul (de 12 países pesquisados), duas são provenientes da América do Norte (de três países pesquisados) e duas da América Central (de 21 países pesquisados).

Os órgãos nacionais de saúde do Canadá, Chile, Colômbia, EUA e Jamaica apresentaram mais de um documento com diretrizes, abordando a promoção de AF em certas especificidades, como: grupos de gestantes (Canadá) ${ }^{10}$, mulheres perinatais $(\text { Chile })^{14}$, grupos de pessoas com doenças crônicas não-transmissíveis (Chile $)^{13}$, grupos de pessoas com esclerose múltipla (Canadá) ${ }^{10}$, no ambiente de trabalho (Colômbia e Jamaica) ${ }^{17,24} \mathrm{e}$ no contexto do deslocamento (Colômbia) ${ }^{18}$. Por ano de publicação, as diretrizes variaram entre 2011 e 2018.

No Quadro 2, observa-se grande variabilidade em relação aos tópicos abordados pelas diretrizes nacionais de AF incluídas. Em todos os documentos foram apresentadas "abordagens e estratégias para a AF na atenção primária à saúde", "recomendações de AF para a saúde" e o "envolvimento de distintos domínios da AF”. Em contrapartida, apenas a diretriz da Argentina ${ }^{11}$ apresentou "abordagens e estratégias para a AF na atenção secundária”.

Excetuando-se o Chile, todos os outros países trabalharam com "conceitos de atividade física". Porém, somente três países apresentaram diretrizes que abordassem o ambiente construído e infraestrutura (Colômbia ${ }^{15,18}$, Costa Rica ${ }^{19}$ e Estados Unidos ${ }^{21,22}$ ).

Quanto aos “correlatos e determinantes”, seis países abordaram este tema nas diretrizes (Canadá ${ }^{10}$, Colômbia ${ }^{15}$, Costa Rica ${ }^{19}$, Equador ${ }^{20}$, Jamai$\mathrm{Ca}^{23}$ e Peru $\left.{ }^{25}\right)$. Quanto aos temas que se relacionam às intervenções para a promoção da $\mathrm{AF}$, seis países $(60 \%)$ continham ações em cada um dos itens como "orientações para ações e políticas" (Argentina $^{11}$, Colômbia ${ }^{15}$, Costa Rica ${ }^{19}$, Estados Unidos $^{21,22}$, Jamaica ${ }^{23}$ e Peru ${ }^{25}$ ) e "ações intersetoriais" (Argentina ${ }^{11}$, Colômbia ${ }^{15}$, Costa Rica ${ }^{19}$, Equador $^{20}$, Estados Unidos ${ }^{21,22}$ e Peru ${ }^{25}$ ). 
Quadro 1. Informações gerais sobre as diretrizes nacionais de atividade física do continente americano.

\begin{tabular}{|l|l|}
\hline \multicolumn{1}{|c|}{ País } & \multicolumn{1}{c|}{ Título da diretriz (ano de publicação) } \\
\hline Argentina & Manual director de actividad física y salud de la República Argentina $(2016)^{11}$ \\
\hline Canadá & $\begin{array}{l}\text { Canadian 24-hour movement guidelines: an integration of physical activity, sedentary } \\
\text { behaviour, and sleep }(2017)^{10}\end{array}$ \\
\hline Chile & $\begin{array}{l}\text { Recomendaciones para la práctica de actividad física según curso de vida }(2017)^{12} \\
\text { Recomendaciones de actividad física para personas concomorbilidad }(2017)^{13} \\
\text { Guía para el uso de videos de ejercicios y actividad perinatal (nd })^{14}\end{array}$ \\
\hline Colômbia & $\begin{array}{l}\text { Orientaciones para la gestión territorial de la promoción de actividad física }(2018)^{15} \\
\text { ABECÉ actividad física para la salud }(2015)^{16} \\
\text { ABECÉ Actividad física en el entorno laboral }(2015)^{17} \\
\text { Orientación técnica para la promoción del transporte activo }(2014)^{18}\end{array}$ \\
\hline Costa Rica & Plan nacional de actividad física y salud 2011-2021 $(2011)^{19}$ \\
\hline Equador & Guía de actividad física dirigida al personal de salud II $(2011)^{20}$ \\
\hline EUA & $\begin{array}{l}\text { Physical activity guidelines for Americans }-2^{\text {nd }} \text { ed. }(2018)^{21} \\
\text { National physical activity plan }(2016)^{22}\end{array}$ \\
\hline Jamaica & $\begin{array}{l}\text { Physical activity basic resource guide }(\text { nd })^{23} \\
\text { Physical activity guide and toolkit for the workplace }(2015)^{24}\end{array}$ \\
\hline Peru & Gestión para la promoción de la actividad física para la salud $(2015)^{25}$ \\
\hline Uruguai & ¡A MOVERSE! Guía de actividad física $(2017)^{26}$ \\
\hline
\end{tabular}

Fonte: Elaborado pelos autores.

Quadro 2. Tópicos abordados pelas diretrizes nacionais de atividade física do continente americano.

\begin{tabular}{|c|c|c|c|c|c|c|c|c|c|c|}
\hline & Arg & Can & $\mathrm{Chi}^{\mathbf{a}}$ & $\mathrm{Col}^{\mathrm{a}}$ & CR & Equ & EUA $^{\mathbf{a}}$ & $\mathrm{Jam}^{\mathrm{a}}$ & Per & Uru \\
\hline Conceitos e definições de AF & $\mathrm{x}$ & $\mathrm{x}$ & & $\mathrm{x}$ & $\mathrm{x}$ & $\mathrm{x}$ & $\mathrm{x}$ & $\mathrm{x}$ & $\mathrm{x}$ & $\mathrm{x}$ \\
\hline Correlatos e determinantes da AF & & $\mathrm{x}$ & & $\mathrm{x}$ & $\mathrm{x}$ & $\mathrm{x}$ & & $\mathrm{x}$ & $\mathrm{x}$ & \\
\hline $\begin{array}{l}\text { Abordagens e estratégias para a AF na } \\
\text { atenção primária }\end{array}$ & $\mathrm{x}$ & $\mathrm{x}$ & $\mathrm{x}$ & $\mathrm{x}$ & $\mathrm{x}$ & $\mathrm{x}$ & $\mathrm{x}$ & $\mathrm{x}$ & $\mathrm{x}$ & $\mathrm{x}$ \\
\hline $\begin{array}{l}\text { Abordagens e estratégias para a AF na } \\
\text { atenção secundária }\end{array}$ & $\mathrm{x}$ & & & & & & & & & \\
\hline $\begin{array}{l}\text { Abordagens e estratégias para a AF na } \\
\text { atenção terciária }\end{array}$ & $\mathrm{x}$ & $\mathrm{x}$ & & $\mathrm{x}$ & & & $\mathrm{x}$ & $\mathrm{x}$ & & \\
\hline Recomendações de AF para a saúde & $\mathrm{x}$ & $\mathrm{x}$ & $\mathrm{x}$ & $\mathrm{x}$ & $\mathrm{x}$ & $\mathrm{x}$ & $\mathrm{x}$ & $\mathrm{x}$ & $\mathrm{x}$ & $\mathrm{x}$ \\
\hline Envolvimento de distintos domínios da AF & $\mathrm{x}$ & $\mathrm{x}$ & $\mathrm{x}$ & $\mathrm{x}$ & $\mathrm{x}$ & $\mathrm{x}$ & $\mathrm{x}$ & $\mathrm{x}$ & $\mathrm{x}$ & $\mathrm{x}$ \\
\hline $\begin{array}{l}\text { Ambiente construído e infraestrutura para a } \\
\text { prática de AF }\end{array}$ & & & & $\mathrm{x}$ & $\mathrm{x}$ & & $\mathrm{x}$ & & & \\
\hline Orientações para ações e políticas de AF & $\mathrm{x}$ & & & $\mathrm{x}$ & $\mathrm{x}$ & & $\mathrm{x}$ & $\mathrm{x}$ & $\mathrm{x}$ & \\
\hline Ações intersetoriais para a promoção da $\mathrm{AF}$ & $\mathrm{x}$ & & & $\mathrm{x}$ & $\mathrm{x}$ & $\mathrm{x}$ & $\mathrm{x}$ & & $\mathrm{x}$ & \\
\hline Ações de monitoramento e vigilância de $\mathrm{AF}$ & $\mathrm{x}$ & & & $\mathrm{x}$ & $\mathrm{x}$ & & $\mathrm{x}$ & $\mathrm{x}$ & & \\
\hline
\end{tabular}

Legendas: a: foram consideradas nesta síntese todas as diretrizes destes países; Arg: Argentina; Can: Canadá; Chi: Chile; Col: Colômbia; CR: Costa Rica; Que: Equador; EUA: Estados Unidos da América; Jam: Jamaica; Per: Peru; Uru: Uruguai.

Fonte: Elaborado pelos autores.

Metade dos países pesquisados apresentou nas suas diretrizes o tópico de "ações de monitoramento e vigilância” que poderia auxiliar na avaliação dos planos e guias (Argentina ${ }^{11}, \mathrm{Co}^{-}$ lômbia ${ }^{15}$, Costa Rica ${ }^{19}$, Estados Unidos ${ }^{21,22}$ e Jamaica $\left.^{23}\right)$.

\section{Discussão}

A evidência produzida pelo presente estudo destacou que, até maio de 2019, apenas dez dos 36 países do continente americano possuíam diretrizes nacionais de $\operatorname{AF}(27,8 \%)$, sendo a porção central do continente com menor número de 
diretrizes em relação ao número de países componentes (duas diretrizes em 21 países). Complementarmente, mesmo que todas as diretrizes encontradas contenham elementos importantes para a promoção da AF, como "abordagens e estratégias para a atenção primária", "recomendações de AF para a saúde" e orientações para o "envolvimento de distintos domínios da AF", observou-se grande variação em relação aos outros tópicos abordados.

Frente ao levantamento conduzido nos países europeus $^{6}$, a presente síntese sugere um menor percentual de países americanos que possuem diretrizes nacionais de AF chanceladas pelos seus respectivos órgãos de saúde $(27,8 \%$ contra $39,6 \%)$. Entretanto, é prudente que dois fatores sejam levados em conta nesta comparação: 1) as diferenças entre os contextos socioeconômicos e epidemiológicos tanto entre os continentes, quanto entre os próprios países que compõem o continente americano, reconhecendo também, que muitos dos países americanos dispõem de recursos limitados para a área da saúde, o que torna compreensível que a maior parte do seu direcionamento seja conferido à adoção e / ou fortalecimento de estratégias que visam melhorias mais urgentes em indicadores locais (exemplo redução da mortalidade materna e infantil, combate às doenças emergentes, doenças transmitidas por vetores e doenças transmissíveis e, também, aos desastres naturais ${ }^{27,28}$ ) e; 2) o objetivo mais específico do estudo europeu, de identificar a existência de recomendações nacionais de AF, com base em variáveis como frequência, intensidade e duração, sem necessariamente envolver assuntos mais abrangentes sobre a prática de AF.

Cabe mencionar que todos os documentos americanos apresentam recomendações para a $\mathrm{AF}$, com foco nos volumes e intensidades de AF visando melhorias nos indicadores de saúde. Em algumas diretrizes nacionais estas recomendações estão inseridas em um contexto mais amplo de orientações, que preconizam um modo mais ativo de viver ${ }^{10,15,21,26}$. E é nesse sentido que a existência de uma diretriz nacional de AF, elaborada sob o prisma dos seus distintos fatores condicionantes e limitantes, pode enquadrar-se como uma importante estratégia educativa para sua promoção no contexto comunitário, sobretudo em um momento em que são altas as prevalências de pessoas que não atingem as recomendações de AF moderadas e vigorosas nos distintos momentos da vida ${ }^{3}$, assim como uma ferramenta que possa orientar a tomada de decisão por parte dos governos locais.
Da mesma forma, sugere-se que futuras diretrizes nacionais de AF possam extrapolar a visão recomendativa-prescritiva, acampando também evidências e elementos que, além de inserirem e contextualizarem a AF no trabalho ampliado da saúde, possam também empoderar pessoas e instituições enquanto agentes capazes de protegerem a própria saúde e a saúde das pessoasque estão no seu entorno, além de fomentarem ações que sejam capazes de intervir nos determinantes sociais e ambientais da saúde. Logo, pode ser destacada a diretriz nacional canadense $\mathrm{e}^{10}$, que parte de uma perspectiva ampliada com foco no movimento ao longo do dia, integrando também orientações sobre comportamento sedentário e sono.

Em vista de sua emergência na área, vale mencionar que documentos de apenas três países (Colômbia ${ }^{15}$, Costa Rica ${ }^{19}$ e Estados Unidos ${ }^{21,22}$ ) abordaram o ambiente construído e a infraestrutura como parte dos planos e ações. Estudos prévios sugerem que o ambiente construído é um determinante da saúde ${ }^{29-31}$, logo não pode ser desconsiderado nas estratégias de promoção da AF, sobretudo nos países que possuem economias em transição e cidades que crescem sem um maior planejamento. Cabe destacar que algumas cidades latino-americanas, como Bogotá, Cali e São Paulo vêm se reestruturando para melhorar a qualidade de vida das pessoas, com destaque, por exemplo, para a introdução de ciclovias ${ }^{32-35}$.

A existência de uma diretriz nacional de AF não necessariamente reflete (ou ampara) a existência de políticas públicas permanentes para a promoção de AF neste mesmo país e vice-versa. Dessa forma, pode ser mencionado o caso do Brasil, que é pioneiro na introdução de políticas públicas nacionais para a promoção da AF no contexto populacional, desde a metade da década de $2000^{36}$, mas que apenas em 2021 lançou uma diretriz nacional de AF chancelada pelo seu Ministério da Saúde ${ }^{37}$. Vale mencionar que esta diretriz foi lançada quando o presente artigo já estava aceito, no prelo.

O presente estudo possui algumas limitações, como, por exemplo a especificidade por diretrizes chanceladas pelos órgãos nacionais do setor saúde. Esta decisão foi tomada partindo da premissa que o setor saúde é o mais relacionado à promoção da $\mathrm{AF}$, mas não o único, em vista do seu potencial no debate de outros setores, como esporte, lazer, educação, ambiente, infraestrutura e transportes. Também, é prudente esclarecer que não fora objetivo do presente artigo sumarizar as políticas, planos e / ou ações voltadas a promoção da AF nos países americanos, tal como se fez 
em um estudo prévio ${ }^{37}$, que aponta a carência de políticas nos países de baixa e média renda. Nesse sentido, pode-se mencionar que, para um melhor efeito das ações institucionais voltadas à promoção da AF, estas devem estar inseridas em um contínuo de políticas públicas e ações de vigilância. O estudo de Sallis et al. aponta que muitos avanços foram feitos em relação à vigilância da AF no contexto global. Porém, apesar do aumento do número de políticas, ações e planos em países das Américas entre 2010 a 2013, houve um decréscimo no biênio posterior (2013-2015)3.

Por fim, a evidência apresentada no presente estudo permite concluir que poucos países do continente americano apresentam diretrizes para a $\mathrm{AF}$, e que no conjunto disponível de diretrizes ainda se percebe grande ênfase no enfoque recomendativo-prescritivo, desconsiderando importantes questões contextuais, ambientais e de vigilância. Dessa forma, ponderando-se pela situação epidemiológica, disponibilidade de recursos e prioridades de saúde entre os países americanos, recomenda-se que novas diretrizes nacionais sejam formuladas, fundamentadas na complexidade dos determinantes e consequências da AF, no sentido de alcançar uma melhor orientação e incentivo de políticas de promoção da AF no contexto populacional.

\section{Colaboradores}

PH Guerra e AA Florindo responderam pela concepção da ideia central, planejamento, busca e identificação das diretrizes, análise e interpretação das informações e redação do texto. LMT Garcia, EHC Ribeiro e ALR Maretti fizeram o planejamento, busca e identificação das diretrizes, análise e interpretação das informações e revisão do texto. DR Andrade fez o planejamento, busca e identificação das diretrizes, contato com pesquisadores externos, análise e interpretação das informações e revisão do texto. 


\section{Referências}

1. World Health Organization (WHO). WHO Global Strategy on Diet, Physical Activity and Health. Geneva: WHO; 2004.

2. World Health Organization (WHO). Global action plan on physical activity 2018-2030: more active people for a healthier world. Geneva: WHO; 2018.

3. Sallis JF, Bull F, Guthold R, Heath GW, Inoue S, Kelly P, Oyeyemi AL, Perez LG, Richards J, Hallal PC. Lancet Physical Activity Series 2 Executive Committee. Progress in physical activity over the Olympic quadrennium. Lancet 2016; 388(10051):1325-1336.

4. World Health Organization (WHO). Global recommendations on physical activity for health. Geneva: WHO; 2010.

5. Brasil. Ministério da Saúde (MS). Guia alimentar para a população brasileira. Brasília: MS; 2014.

6. Kahlmeier S, Wijnhoven TM, Alpiger P, Schweizer C, Breda J, Martin BW. National physical activity recommendations: systematic overview and analysis of the situation in European countries. BMC Public Health 2015; 15:133.

7. Oja P, Bull FC, Fogelholm M, Martin BW. Physical activity recommendations for health: what should Europe do? BMC Public Health 2010;10:10.

8. Pate RR, Pratt M, Blair SN, Haskell WL, Macera CA, Bouchard C, Buchner D, Ettinger W, Heath GW, King AC, Kriska A, Leon AS, Marcus BH, Morris J, Paffenbarger Jr RS, Patrick K, Pollock ML, Rippe JM, Sallis J, Wilmore JH. Physical activity and public health. A recommendation from the Centers for Disease Control and Prevention and the American College of Sports Medicine. JAMA1995; 273(5):402-407.

9. Australia. Department of Health. Australia's physical activity and sedentary behaviour guidelines and the australian 24-hour movement guidelines. [cited 2020 Apr 10]. Available from: https://www1.health.gov.au/ internet/main/publishing.nsf/Content/health-pubhlth-strateg-phys-act-guidelines.

10. Canada. Canadian Society for Exercise Physiology. Canadian 24-hour movement guidelines: an integration of physical activity, sedentary behaviour, and sleep. [cited 2020 Apr 10]. Available from: https://csepguidelines.ca/.

11. Argentina. Ministerio de Salud de la Nación. Manual director de actividad física y salud de la República Argentina. Buenos Aires: 2016. [acceso 2020 Abr 10]. Disponible en: http://www.msal.gob.ar/images/stories/bes/graficos/0000000074cnt-manual-actividadfisica_2017.pdf

12. Chile. Ministerio del Deporte; Ministerio de Salud; Ministerio de Educación. Recomendaciones para la práctica de actividad física según curso de vida. Santiago de Chile: 2017. [acceso 2020 Abr 10]. Disponible en: http://www.mindep.cl/wp-content/uploads/2016/06/ Recomendaci\%C3\%B3n-para-la-pr\%C3\%A1cticade-actividad-f\%C3\%ADsica-seg\%C3\%BAn-cursode-vida.pdf

13. Chile. Ministerio de Salud. Recomendaciones de actividad física para personas concomorbilidad. Santiago de Chile: 2017. [acceso 2020 Abr 10]. Disponible en: http://www.repositoriodigital.minsal.cl/handle/2015/ 872
14. Ministerio de Salud (Chile). Guía para el uso de videos de ejercicios y actividad perinatal. Santiago de Chile. [acceso 2020 Abr 10]. Disponible en: http://www.crececontigo.gob.cl/wp-content/uploads/2019/04/Guiade-ejercicios-perinatal-web.pdf

15. Colômbia. Ministerio de Salud y Protección Social. Orientaciones para la gestión territorial de la promoción de la actividad física. Bogotá: 2018. [acceso 2020 Abr 10]. Disponible en: https://www.minsalud.gov.co/ sites/rid/Lists/BibliotecaDigital/RIDE/VS/PP/ENT/ orientaciones-gestion-territorial-promocion-actvidad -fisica.pdf

16. Colômbia.Ministerio de Salud y Protección Social ABECÉ Actividad Física para la Salud. Bogotá: 2015 [acceso 2020 Abr 10]. Disponible en: https://www. minsalud.gov.co/sites/rid/Lists/BibliotecaDigital/ RIDE/VS/PP/ENT/abece-actividad-fisica-para-la-salud.pdf

17. Colômbia. Ministerio de Salud y ProtecciónSocial ABECÉ Actividad física en el entorno Laboral. Bogotá: 2015. [acceso 2020 Abr 10]. Disponible en: https:// www.minsalud.gov.co/sites/rid/Lists/BibliotecaDigital/RIDE/VS/PP/ENT/abece-entorno-laboral.pdf

18. Colômbia. Ministerio de Salud y Protección Social. Orientación técnica para la promocióndel transporte activo. Bogotá: 2014. [acceso 2020 Abr 10]. Disponible en: https://www.minsalud.gov.co/sites/rid/Lists/ BibliotecaDigital/RIDE/VS/PP/ENT/Orientacionestecnicas-promocion-transporte-activo.pdf

19. Costa Rica. Ministerio de Salud; Ministerio de Deporte y Recreación. Plan Nacional de Actividad Física y Salud 2011-2021. San José: 2011. [acceso 2020 Abr 10]. Disponible en: https://www.ministeriodesalud. go.cr/index.php/biblioteca-de-archivos/sobre-elministerio/politcas-y-planes-en-salud/planes-ensalud/1164-plan-nacional-de-actividad-fisica- $y$-salud-2011-2021/file

20. Equador. Ministerio de Salud Pública. Guía de actividad física dirigida al personal de salud II. Guayaquil: 2011. [acceso 2020 Abr 10]. Disponible en: https:// aplicaciones.msp.gob.ec/salud/archivosdigitales/ documentosDirecciones/dnn/archivos/GUIA $\% 20$ DE\%20ACTIVIDAD\%20FISICA\%202.pdf

21. Estados Unidos da América. United States Department of Health and Human Services. Physical activity guidelines for americans. 2nd edition. Washington: 2018. [cited 2020 Apr 10]. Available from: https:// health.gov/sites/default/files/2019-09/Physical_Activity_Guidelines_2nd_edition.pdf

22. Estados Unidos da América. National Physical Activity Plan Alliance. National physical activity plan Columbia: 2016. [cited 2020 Apr 10]. Available from: https://www.physicalactivityplan.org/index.html

23. Jamaica. Ministry of Health. Physical activity basic resource guide. Kingston: 2016. [cited 2020 Apr 10]. Available from: https://moh.gov.jm/wp-content/ uploads/2016/07/Physical-Activity-Basics-for-HEO5-min.pdf

24. Jamaica. Ministry of Health. Physical activity guide and toolkit for the workplace. Kingston: 2015. [cited 2020 Apr 10]. Available from: https://www.moh.gov. jm/wp-content/uploads/2016/07/Physical-ActivityGuide-for-the-Workplace-final-min.pdf 
25. Peru. Ministerio de Salud. Documento técnico: Gestión para la promoción de la actividad física para la salud. Lima: 2015. [acceso 2020 Abr 10]. Disponible en: http://bvs.minsa.gob.pe/local/MINSA/3299.pdf

26. Uruguai. Ministerio de Salud; Secretaria Nacional del Deporte. ¡A MOVERSE! Guía de actividad física. Montevidéu: 2017. [acceso 2020 Abr 10]. Disponible en:https://uruguayeduca.anep.edu.uy/recursos-educativos/2276

27. Organização Pan-Americana da Saúde (OPAS). Saúde nas Américas+, Edição de 2017. Resumo do panorama regional e perfil do Brasil. Washington, D.C.: OPAS; 2017

28. Pan American Health Organization (PAHO). Evidencia e Inteligencia para la AcciónenSalud / Análisis de Salud, Métricas y Evidencia: Situación de Saludenlas Américas: Indicadores Básicos 2018. Washington, D.C.: PAHO; 2018.

29. Travert AS, Sidney Annerstedt K, Daivadanam M. Built Environment and Health Behaviors: Deconstructing the Black Box of Interactions-A Review of Reviews. Int J Environ Res Public Health 2019; 16(8):E1454.

30. Groot R, van den Hurk K, Schoonmade LJ, Kort WLAM, Brug J, Lakerveld J. Urban-rural differences in the association between blood lipids and characteristics of the built environment: a systematic review and meta-analysis. BMJ Glob Health 2019; 4(1):e001017.

31. Chandrabose M, Rachele JN, Gunn L, Kavanagh A, Owen N, Turrell G, Giles-Corti B, Sugiyama T. Built environment and cardio-metabolic health: systematic review and meta-analysis of longitudinal studies. Obes Rev 2019; 20(1):41-54.

32. Torres A, Sarmiento OL, Stauber C, Zarama R. The Ciclovia and Cicloruta programs: promising interventions to promote physical activity and social capital in Bogotá, Colombia. Am J Public Health 2013; 103(2):e23-30.

33. Gómez LF, Mosquera J, Gómez OL, Moreno J, Pinzon JD, Jacoby E, Cepeda M, Parra DC. Social conditions and urban environment associated with participation in the Ciclovia program among adults from Cali, Colombia. Cad Saude Publica 2015; 31(Suppl. 1):257266.
34. Florindo AA, Barrozo LV, Turrell G, Barbosa JPDAS, Cabral-Miranda W, Cesar CLG, Goldbaum M. Cycling for Transportation in Sao Paulo City: Associations with Bike Paths, Train and Subway Stations. Int $J$ Environ Res Public Health 2018; 15(4):E562.

35. Florindo AA, Barrozo LV, Cabral-Miranda W, Rodrigues EQ, Turrell G, Goldbaum M, Cesar CLG, Giles-Corti B. Public Open Spaces and Leisure-Time Walking in Brazilian Adults. Int J Environ Res Public Health 2017;14(6):E553.

36. Loch MR, Knuth AG, Silva ICM, Guerra PH. As práticas corporais/atividade física nos 30 anos do Sistema Único de Saúde. Cien Saude Colet 2018; 23(10):3469.

37. Klepac Pogrmilovic B, O’Sullivan G, Milton K, Biddle SJH, Bauman A, Bull F, Kahlmeier S, Pratt M, Pedisic Z.A global systematic scoping review of studies analysing indicators, development, and content of national-level physical activity and sedentary behaviour policies. Int J BehavNutr Phys Act 2018; 15(1):123.

Artigo apresentado em 03/04/2020

Aprovado em 09/06/2020

Versão final apresentada em 11/06/2020

Editores-chefes: Romeu Gomes, Antônio Augusto Moura da Silva 